Canadian

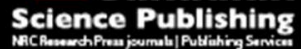

Canadian Journal of Physiology and Pharmacology Revue canadienne de physiologie et pharmacologie

\title{
Irisin levels in relation to metabolic and liver functions in Egyptian patients with metabolic syndrome
}

\begin{tabular}{|r|l|}
\hline Journal: & Canadian Journal of Physiology and Pharmacology \\
\hline Manuscript ID & cjpp-2015-0371.R1 \\
\hline Manuscript Type: & Article \\
\hline Date Submitted by the Author: & $15-$ Sep-2015 \\
\hline Complete List of Authors: & $\begin{array}{l}\text { Rizk, Fatma; Faculty of medicine, Tanta university, Physiology department } \\
\text { Elshweikh, Samah; Tanta university, internal medicine department } \\
\text { Abd El-Naby, Amira; Faculty of Medicine, Tanta University, clinical } \\
\text { pathology depatment }\end{array}$ \\
\hline Keyword: & Irisin, Metabolic syndrome, Fatty liver. \\
\hline \multicolumn{2}{|c}{} \\
\hline
\end{tabular}


Irisin levels in relation to metabolic and liver functions in Egyptian patients with metabolic syndrome

Fatma H. Rizk ${ }^{\text {a, }}$, Samah A. Elshweikh ${ }^{\text {b }}$, Amira Y. Abd El-Naby ${ }^{\text {c }}$

Departments of a Physiology, ${ }^{\mathrm{b}}$ Internal Medicine, ${ }^{\mathrm{c}}$ Clinical Pathology,

Faculty of Medicine, Tanta University, Egypt.

"Corresponding author, Egypt, +2- 01064122556,

e-mail Fatma.rezk@med.tanta.edu.eg 


\section{Abstract}

Irisin is a new myokine that is suspected to influence metabolic syndrome (MetS). However, there is a great controversy as regard its level in cases of MetS and its correlation with different metabolic parameters. The aim of the present study was to assess irisin levels in MetS patients and study its relationship to metabolic and liver functions to evaluate the possible role of the liver in regulation of this level. 60 subjects included in this experiment and divided into three groups: Group I (normal control persons), group II (MetS patients with normal liver enzymes) and group III (MetS with elevated liver enzymes and fatty liver). Serum irisin levels showed significant increases in group II and III compared with group I and significant increases in group III compared with group II. Also, irisin levels were positively correlated with body mass index, serum triglycerides, homeostatic model assessment of insulin resistance index (HOMA-IR), and liver enzymes. We concluded that serum irisin level increased in patients with MetS especially those with elevated liver enzymes and had a positive correlation with parameters of lipid metabolism and glucose homeostasis with the possibility of hepatic clearance to irisin.

Keywords: Irisin, Metabolic syndrome, Fatty liver. 


\section{Introduction}

The metabolic syndrome (MetS) is a combination of medical disorders as it includes obesity, insulin resistance, hypertension, hypertriglyceridemia, and low high density lipoprotein cholesterol (HDL- C) and it may be associated with a number of other conditions including non-alcoholic fatty liver disease (NAFLD), chronic kidney disease, endothelial dysfunction, and systemic inflammation. These signs are the primary precursor of the development of type 2 diabetes and cardiovascular diseases (CVD) (Ronis et al. 2012). Various metabolic hormones influence obesity and its associated complications. Among those hormones, the adipokines which secreted by adipose tissue and provide a link between obesity, metabolic syndrome and its associated complications (Jung and Choi 2014). Besides adipokines, myokines, which are myocyte secreted proteins, have recently been implicated in the pathogenesis of obesity and its associated metabolic and vascular complications. Among those, irisin which is a new myokine that is suspected to reverse visceral obesity and improve blood glucose level

\section{(Boström et al. 2012).}

Irisin is a hormone whose expression is induced by exercise and peroxisome proliferator-activated receptor- $\gamma$ coactivator $1 \alpha(\mathrm{PGC} 1 \alpha)$. Previous studies reported that it acts on white adipose cells leading to brown-fat-like 
development (Boström et al. 2012). As irisin upregulates the expression of uncoupling protein-1 (a regulator of thermogenesis in brown fat) (Zhang et al. 2014). This conversion of white adipocytes to brown adipocytes leads to increase in energy expenditure and thermogenesis with subsequent improvement of insulin sensitivity, reductions in body weight, and improved glucose tolerance in mice (Fisher et al. 2012).

So irisin level suspected to be related to different metabolic parameters. However, there is a great controversy as regard its level in cases of metabolic syndrome and its correlation with different metabolic parameters between different populations (Chen et al. 2015). As Park et al. (2013) reported that irisin levels are significantly increased in patients with metabolic syndrome as compared to subjects without metabolic syndrome. While, Yan et al. (2014) stated that irisin levels are significantly decreased in patients with MetS as compared to controls. Moreover, the correlation between irisin and the metabolic parameters showed a great discrepancy as Pardo et al. (2014) reported that irisin is positively correlated with BMI while, Choi et al. (2013) stated that irisin is negatively correlated with BMI but, Sanchis-Gomar et al. (2014) didn't find a positive or negative correlation between irisin level and BMI. 
Also the regulation of irisin level in humans is not clearly explained as Ebert et al.( 2014) showed that irisin is not eliminated by the kidney and the possible clearance of irisin by the liver should be evaluated in future studies. Therefore, further studies are warranted to address this discrepancy on the level of irisin in MetS in different populations and the role of liver in regulation of it.

In this study, we will measure the level of irisin in Egyptian patients with metabolic syndrome and assess the association between irisin levels and different metabolic parameters and liver enzymes to evaluate the possible role of the liver in regulation of this level. 


\section{Subjects and methods}

\section{Subjects}

This cross-sectional study was carried out on 60 subjects (26 male and 34 female) divided into three groups. Twenty healthy subjects (10 male and 10 female) were included in the study as a normal control group (group I). Twenty patients ( 7 male and 13 female) with metabolic syndrome and normal liver enzymes (group II) and twenty patients (9 male and 11 female) with metabolic syndrome and elevated liver enzymes, fatty liver disease to study the possible role of liver in regulation of irisin level (group III). They were randomly selected from outpatients of the Endocrinology Unit, Internal Medicine Department, Faculty of Medicine, Tanta University, from February 2015 to July 2015. Participation in the study was voluntary after written informed consent was obtained from the subjects before the study. As part of the informed consent process, participants' rights as research subjects, privacy of participants, confidentiality of data, the possible risks and benefits were clearly explained.

End-stage malignant disease, acute generalized inflammation, acute infectious disease, viral hepatitis, history of myocardial infarction, an active 
diagnosis of diabetes, dialysis, history of drug abuse and alcohol intake were excluded from our study. All patients aged more than 18 years and the females were non-pregnant and categorized as metabolic syndrome according to WHO criteria (1999) which require the presence of impaired fasting blood glucose or Insulin resistance, Dyslipidemia: triglycerides (TG): $\geq 1.695 \mathrm{mmol} / \mathrm{L}(150 \mathrm{mg} / \mathrm{dl})$ and body mass index $(\mathrm{BMI})>30 \mathrm{~kg} / \mathrm{m} 2$ (Grundy et al. 2004).

\section{Methods}

All patients included in the study were subjected to full medical history, and complete clinical examination. We calculated BMI using the following formula: subject's weight in $\mathrm{kg} /$ height squared in meter (kilogram per square meter). Abdominal ultrasonography examination was done to assess the liver condition. The diagnosis of NAFLD is based on a history of no alcohol intake and the presence of hepatic steatosis by imaging, and the ruling out of other hepatic diseases.

\section{Blood sampling \& biochemical analysis:}

In all subjects, fasting blood samples were collected in the morning after 12 hours fasting for separation of the serum within 30 to 60 minutes of 
collection and stored at $-80^{\circ} \mathrm{C}$. Serum triglycerides (TGS) were measured by GPO enzymatic method described by Fossati and Prencipe (1982), fasting blood glucose levels were measured using enzymatic glucose kits according to the method described by Tietz (1986), serum insulin levels were determined using ELISA kits (Life Technologies, Invitrogen, Camarillo, CA, USA) (cat.No. KAQ1251) in which it is a solid phase sandwich enzyme-linked immunosorbent assay (ELISA). Then we calculate homeostatic model assessment of insulin resistance index (HOMA-IR) Based on seum insulin level $(\mu \mathrm{IU} / \mathrm{ml})$ and serum glucose level $(\mathrm{mg} / \mathrm{dl})$ according to the formula described by Matthews et al. (1985) as HOMA- IR $=$ fasting serum glucose $(\mathrm{mg} / \mathrm{dl}) \mathrm{x}$ fasting serum insulin $(\mu \mathrm{IU} / \mathrm{ml}) / 405$ and The HOMA- IR value of 2.5 is taken as an indicator of IR, serum alanine aminotransferase (ALT) \& serum aspartate aminotransferase (AST) were measured based on the principle of Karmen method (Krefetz and McMillin 2005), serum irisin levels were determined by ELISA kits (BioVendor, Bmo, Czech republic) (Cat. No.: RAG018R) which is a competitive Enzyme Linked-Immunosorbent Assay (ELISA) for quantitative determination of serum irisin in human with reference range $(20-200 \mathrm{ng} / \mathrm{ml})$.

\section{Statistical analysis}


The data were expressed as the mean \pm standard deviation (SD). Statistical comparison between different groups was carried out by using one-way ANOVA. Pearson's correlations were used to test for the associations of irisin with the different variables. All the analyses were performed using Graph Pad Instat, 32 bit for win 95/NT (Version 3.05). $P$-values $<0.05$ were considered statistically significant. 


\section{Results}

\section{Characteristics of study participants}

The characteristics of study subjects are presented in Table (1) and Figure (1). Notably, the mean of age of studied groups was (44.25 \pm 10.46 , $45.45 \pm 8.30$ and $47.85 \pm 6.24)$ respectively which was not significantly different between the studied groups. While, BMI $(32.54 \pm 1.80$ and $35.91 \pm 3.00$ VS $22.85 \pm 1.68)$, serum triglycerides $(170.05 \pm 12.86$ and 193.8 \pm 12.06 VS $110.35 \pm 17.97)$, fasting blood glucose (115.45 \pm 2.41 and $117.38 \pm 1.86$ VS $82.6 \pm 9.30)$, serum insulin $(20.90 \pm 3.20$ and $21.32 \pm 3.08$ VS $12.63 \pm 1.27)$, HOMA- IR (2.78 \pm 0.41 and $2.87 \pm 0.40 \mathrm{VS} 1.59 \pm 0.20), \quad$ ALT (30.23 \pm 6.41 and $64.88 \pm 9.09$ VS 27.97 \pm 5.97$)$, AST $(22.77 \pm 6.94$ and $52.05 \pm 7.17$ VS $21.81 \pm 7.09)$, and irisin (194.46 \pm 9.85 and $209.32 \pm 26.80$ VS $139.77 \pm 13.23$ ) in patients of group II and group III respectively were statistically significant $(P<0.05)$ as compared with normal control group. Also, serum triglycerides, ALT, AST, and irisin were statistically significant $(P<0.05)$ in patients of group III as compared with patients of group II.

Correlation between serum irisin and measured variables in patients with metabolic syndrome and normal liver enzymes 
Pearson's correlations analysis showed that serum irisin was positively correlated with BMI $(r=0.639, p=0.002)$, serum triglycerides $(r=0.457$, $p=0.043)$, HOMA-IR $(r=0.498, p=0.026)$, ALT $(r=0.473, p=0.035)$ and $\operatorname{AST}(r=0.455, p=0.044)$ Table (2).

Correlation between serum irisin and measured variables in patients with metabolic syndrome and elevated liver enzymes

Pearson's correlations analysis showed that serum irisin was positively correlated with BMI $(r=0.612, p=0.004)$, serum triglycerides $(r=0.625$, $p=0.003)$, HOMA-IR $(r=0.473, p=0.035)$ ALT $(r=0.524, p=0.018)$ and AST $(r=0.452, p=0.045)$ Table (3) and Figure (2). 


\section{Discussion}

Irisin is a recently known hormone that has been suggested to play an important role in energy homeostasis and obesity (Boström et al. 2012). Numerous studies focus on the association of irisin with MetS but still there are great discrepancies as regard its level and its association with different metabolic parameters in MetS (Chen et al. 2015). Also, the regulation of irisin level in the body still needed to be investigated (Ebert et al. 2014). So, in this study we assessed irisin levels in patients with MetS with and without elevated liver enzymes and correlated irisin levels with different metabolic parameters and liver enzymes to assess the role of irisin in lipid and glucose homeostasis and to investigate the possibility of hepatic regulation of irisin level.

The results of the present work showed significant increases in serum irisin levels of all patients with metabolic syndrome especially those with elevated liver enzymes as compared with normal subjects. These results were in agreement with Park et al. (2013) who stated that baseline irisin levels are significantly increased in patients with metabolic syndrome than in subjects without metabolic syndrome. While Yan et al. (2014) showed that circulating irisin levels are significantly decreased in subjects with MetS 
compared to controls. This may be due to the different populations included in these studies, as the study of Park et al included patients with MetS with high BMI making it the major factor, but the study from Yan et al recruited patients with central obesity making glucose homeostasis and insulin resistance (IR) the most important factors (Chen et al. 2015). The mechanism of increased irisin level in MetS still not clear but it may be due to increased secretion of irisin by the increased mass of adipose and muscle tissues in obesity (Park et al. 2013). This is supported by the results of the present work which showed a positive correlation between irisin and BMI in patients with metabolic syndrome and in patients with metabolic syndrome associated with elevated liver enzymes. Also, it may be a compensatory increase of irisin level to overcome obesity and the MetS (Yan et al. 2014). In pathological conditions of obesity, physiological irisin levels cannot maintain the balance of energy storage and expenditure, and excess irisin is secreted from skeletal muscle, and even adipose tissue to compensate for increased fat storage in the $\operatorname{body}($ Chen et al. 2015). This mechanism in accordance with the results of the present work which showed that there was a positive correlation between irisin and TGs levels in patients with metabolic syndrome and in patients with metabolic syndrome associated with elevated liver enzymes. In addition, the results of the present work 
showed that there were significant increases in serum irisin and TGs levels in patients with metabolic syndrome associated with elevated liver enzymes and fatty liver as compared to patients with metabolic syndrome. These results raise the possibility of the role of irisin in lipid metabolism and the development of non-alcoholic fatty liver disease (NALFD), and the mechanisms that clarify this role need further studies (Chen et al. 2015). Furthermore, the increased irisin levels in MetS may be due to the presence of irisin resistance, similar in a way to insulin and leptin resistance in MetS (Zhang and Scarpace 2006). This mechanism supported by our study which showed that there was a positive correlation between irisin and HOMA-IR in patients with metabolic syndrome and in patients with metabolic syndrome associated with elevated liver enzymes but this hypothesis need to be tested in future studies by studying the nature of irisin receptors. The positive correlation between irisin and HOMA-IR in patients with metabolic syndrome may reflect the role of irisin in glucose tolerance which explained by Zhang and Scarpace ( 2006) who stated that irisin promotes the expression of betatrophin hormone which in turn promotes insulin secretion by increasing the proliferation of pancreatic $\beta$-cell with subsequent improvement of glucose tolerance. In contrast to our results AlDaghri et al. (2013) stated that circulating irisin level was negatively 
correlated with HOMA-IR in a cross-sectional study of girls aged $12.9 \pm 3.2$ years-old. This difference may be due to the difference in the studied populations as our study included adult patients with MetS and did not include children so the differences in age, sex and included patients may be the cause of this discrepancy in the results.

Moreover, the present study revealed that there was a positive correlation between serum irisin level and liver enzymes in patients with metabolic syndrome and in patients with metabolic syndrome associated with elevated liver enzymes. This indicates the possible influence of hepatic clearance on circulating irisin level. This hypothesis needs to be tested in future studies to elucidate the underlying mechanism of hepatic clearance of irisin.

This study has some limitations. Our sample size was relatively small. Moreover, our strict patients selection criteria excluded children, pregnant female, patients with end-stage malignant diseases, acute generalized inflammation, acute infectious disease, viral hepatitis, history of myocardial infarction, an active diagnosis of diabetes mellitus, dialysis, history of drug abuse and alcohol intake. Future studies with larger sample sizes and measuring irisin levels in different age groups, different conditions, and populations will be needed.

\section{Conclusion}


Serum irisin levels were significantly increased in patients with metabolic syndrome especially those with elevated liver enzymes and had positive correlation with BMI, serum TGs, HOMA-IR, ALT and AST which might suggest the role of irisin in lipid metabolism and glucose homeostasis with the possibility of irisin clearance by the liver. Future studies will be required to ascertain the precise mechanisms supporting these associations.

\section{Competing interests}

The authors declare that they have no competing interests.

\section{Author contributions}

FHR and SAE designed the study; FHR, SAE, AYA performed the experiment; FHR analyzed the data and wrote the paper; all authors revised the paper and approved the final version of it. 


\section{References}

Al-Daghri, N.M., Alkharfy, K.M., Rahman, S., Amer, O.E., Vinodson, B., Sabico, S., et al. 2013. Irisin as a predictor of glucose metabolism in children: sexually dimorphic effects. Eur. J .Clin. Invest. 44: 119124. doi: 10.1111/eci.12196.

Boström, P., Wu, J., Jedrychowski, M.P., Korde, A., Ye, L., Lo, J.C., et al. 2012. A PGC1-alpha-dependent myokine that drives brown-fat-like development of white fat and thermogenesis. Nature, 481: 463-468. doi:10.1038/nature10777.

Chen, J.Q., Huang, Y.Y., Gusdon, A.M., and Qu, S. 2015. Irisin: a new molecular marker and target in metabolic disorder. Lipids in Health and Disease, 14:2. doi: 10.1186/1476-511X-14-2.

Choi, Y.K., Kim, M.K., Bae, K.H., Seo, H.A., Jeong, J.Y., Lee, W.K., et al. 2013. Serum irisin levels in new-onset type 2 diabetes. Diabetes Res. Clin. Pract. 100 (1): 96-101. doi: 10.1016/j.diabres.2013.01.007.

Ebert, T., Focke, D., Petroff, D., Wurst, U., Richter, J., Bachmann, A., et al. 2014. Serum levels of the myokine irisin in relation to metabolic and 
renal function. Eur. J. Endocrinol. 170 (4): 501-506. doi: 10.1530/EJE-13-1053.

Fisher, F.M., Kleiner, S., Douris, N., Fox, E.C., Mepani, R.J., Verdeguer, F., et al. 2012. FGF21 regulates PGC-1 $\alpha$ and browning of white adipose tissues in adaptive thermogenesis. Genes Dev. 26: 271-281. doi: 10.1101/gad.177857.111.

Fossati, P., and Prencipe, L. 1982. Triglycerides determination after enzymatic hydrolysis. Clin. Chem. 28: 2077-2080.

Grundy, S. M., Brewer, H. B., Cleeman, J. I., Smith, S. C., and Lenfant, C. 2004. Definition of Metabolic Syndrome : Report of the National Heart, Lung, and Blood Institute/American Heart Association Conference on Scientific Issues Related to Definition, Circulation, Vol. 109. pp. 433-438.

Jung, U.J., and Choi, M.S. 2014. Obesity and Its Metabolic Complications: The Role of Adipokines and the Relationship between Obesity, Inflammation, Insulin Resistance, Dyslipidemia and Nonalcoholic Fatty Liver Disease. Int. J. Mol. Sci. 15 (4): 6184-6223. doi: 10.3390/ijms 15046184. 
Krefetz, R.G., and McMillin, G.A. 2005. Enzymes. In Clinical chemistry principles, procedures, correlations. Edited by M.L. Bishop, E.P. Fody, and L. Schoeff (fifth edition ). United States of America, Lippincott Williams \& Wilkins, pp. 250- 251.

Matthews, D.R., Hosker, J.P., Rudenski, A.S., Naylor, B.A., Treacher, D.F., and Turner, R.C. 1985. Homeostasis model assessment: insulin resistance and beta-cell function from fasting plasma glucose and insulin concentrations in man. Diabetologia, 28: 412-419.

Pardo, M., Crujeiras, A.B., Amil, M., Aguera, Z., Jimenez-Murcia, S., Banos, R., et al. 2014. Association of irisin with fat mass, resting energy expenditure, and daily activity in conditions of extreme body mass index. Int. J. Endocrinol. 2014: 1-9. Available from http://dx.doi.org/10.1155/2014/857270.

Park, K.H., Zaichenko, L., Brinkoetter, M., Thakkar, B., Sahin-Efe, A., Joung, K.E., et al. 2013. Circulating irisin in relation to insulin resistance and the metabolic syndrome. J. Clin. Endocrinol. Metab. 98 (12): 4899-907. doi: 10.1210/jc.2013-2373. 
Ronis, M. J. J., Baumgardner, J. N., Marecki, J. C, Wu, L.H.X, Shankar, K., Cleves, M. A., et al. 2012. Dietary fat source alters hepatic gene expression profile and determines the type of liver pathology in rats overfed via total enteral nutrition. Physiol. Genomics, 44: 1073-1089. doi: 10.1152/physiolgenomics.00069.2012.

Sanchis-Gomar, F., Alis, R., Pareja-Galeano, H., Sola, E., Victor, VM., Rocha. M., et al. 2014. Circulating irisin levels are not correlated with BMI, age, and other biological parameters in obese and diabetic patients. Endocrine, 46 (3): 674-7. doi: 10.1007/s12020-014-0170-9.

Tietz, N.W. 1986. Determination of blood glucose, Text book of clinical chemistry. WB Saunders. Co. London, Philadelphia: pp. 796.

Yan, B., Shi, X., Zhang, H., Pan, L., Ma, Z., Liu, S., et al. 2014. Association of serum irisin with metabolic syndrome in obese Chinese adults. PLoS One, 9 (4): e94235. doi: 10.1371/journal.pone.0094235. eCollection 2014.

Zhang, Y. and Scarpace, P.J. 2006. The role of leptin in leptin resistance and obesity. Physiol. Behav. 88: 249-256. PMID: 16782141 
Zhang, Y., Li, R., Meng, Y., Li, S., Donelan, W., Zhao, Y., et al. 2014. Irisin stimulates browning of white adipocytes through mitogen-activated protein kinase p38 MAP kinase and ERK MAP kinase signaling. Diabetes, 63: 514-525. doi: 10.2337/db13-1106. 
Table (1) comparison between the studied groups as regard measured variables

\begin{tabular}{|l|l|l|l|}
\hline parameters & $\begin{array}{l}\text { Group I } \\
(\boldsymbol{n}=\mathbf{2 0})\end{array}$ & $\begin{array}{l}\text { Group II } \\
(\boldsymbol{n}=\mathbf{2 0})\end{array}$ & $\begin{array}{l}\text { Group III } \\
(\boldsymbol{n}=\mathbf{2 0})\end{array}$ \\
\hline Age (years) & $44.25 \pm 10.46$ & $45.45 \pm 8.30$ & $47.85 \pm 6.24$ \\
\hline BMI $\left(\mathrm{kg} / \mathrm{m}^{2}\right)$ & $22.85 \pm 1.68$ & $32.54 \pm 1.80^{\mathrm{a}}$ & $35.91 \pm 3.00^{\mathrm{a}, \mathrm{b}}$ \\
\hline TGS $(\mathrm{mg} / \mathrm{dl})$ & $110.35 \pm 17.97$ & $170.05 \pm 12.86^{\mathrm{a}}$ & $193.8 \pm 12.06^{\mathrm{a}, \mathrm{b}}$ \\
\hline FBG $(\mathrm{mg} / \mathrm{dl})$ & $82.6 \pm 9.30$ & $115.45 \pm 2.41^{\mathrm{a}}$ & $117.38 \pm 1.86^{\mathrm{a}}$ \\
\hline Insulin $(\mu \mathrm{IU} / \mathrm{ml})$ & $12.63 \pm 1.27$ & $20.90 \pm 3.20^{\mathrm{a}}$ & $21.32 \pm 3.08^{\mathrm{a}}$ \\
\hline HOMA-IR & $1.59 \pm 0.20$ & $2.78 \pm 0.41^{\mathrm{a}}$ & $2.87 \pm 0.40^{\mathrm{a}}$ \\
\hline ALT (U/L) & $27.97 \pm 5.97$ & $30.23 \pm 6.41$ & $64.88 \pm 9.09^{\mathrm{a}, \mathrm{b}}$ \\
\hline AST (U/L) & $21.81 \pm 7.09$ & $22.77 \pm 6.94$ & $52.05 \pm 7.17^{\mathrm{a}, \mathrm{b}}$ \\
\hline
\end{tabular}

Data are given as mean $\pm S D .{ }^{a} P<0.05$ vs group $I .{ }^{b} P<0.05$ vs group II. 
Table (2) Correlation between serum irisin and different variables in patients with metabolic syndrome and normal liver enzymes

\begin{tabular}{|l|l|l|}
\hline \multicolumn{1}{|c|}{ Parameters } & \multicolumn{2}{c|}{ Serum irisin (ng/ml) } \\
\cline { 2 - 3 } & \multicolumn{2}{|c|}{$r$} \\
\hline BMI $\left(\mathrm{kg} / \mathrm{m}^{2}\right)$ & 0.639 & $0.002^{*}$ \\
\hline TGS (mg/dl) & 0.457 & $0.043^{*}$ \\
\hline HOMA-IR & 0.498 & $0.026^{*}$ \\
\hline ALT (U/L) & 0.473 & $0.035^{*}$ \\
\hline AST (U/L) & 0.455 & $0.044^{*}$ \\
\hline
\end{tabular}

p $<0.05$ were considered statistically significant. 
Table (3) Correlation between serum irisin and different variables in patients with metabolic syndrome and elevated liver enzymes

\begin{tabular}{|l|l|l|}
\hline \multicolumn{1}{|c|}{ Parameters } & \multicolumn{2}{c|}{ Serum irisin(ng/ml) } \\
\cline { 2 - 3 } & \multicolumn{1}{|c|}{$r$} & \multicolumn{1}{c|}{$p$} \\
\hline BMI $\left(\mathrm{kg} / \mathrm{m}^{2}\right)$ & 0.612 & $0.004^{*}$ \\
\hline TGS (mg/dl) & 0.625 & $0.003^{*}$ \\
\hline HOMA- IR & 0.473 & $0.035^{*}$ \\
\hline AST (U/L) & 0.452 & $0.045^{*}$ \\
\hline
\end{tabular}

p $<0.05$ were considered statistically significant. 
Figure (1) serum irisin levels in studied groups. Data are given as mean \pm SD. ${ }^{a} \boldsymbol{P}<\mathbf{0 . 0 5}$ vs group I. $^{b} \boldsymbol{P}<\mathbf{0 . 0 5}$ vs group II.

Figure (2) Correlation between serum irisin levels and ALT in patients with metabolic syndrome and elevated liver enzymes. $" p<0.05$ were considered statistically significant. 


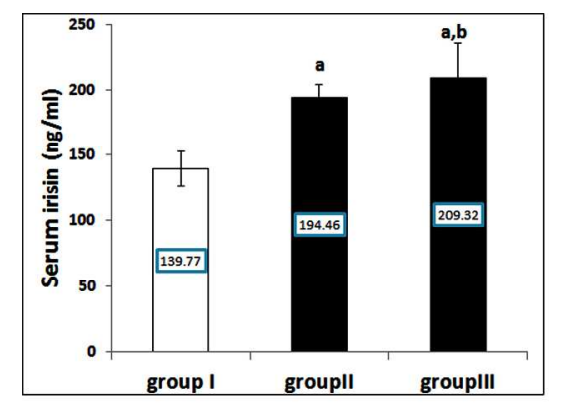

Figure (1) serum irisin levels in studied groups. Data are given as mean $\pm \mathrm{SD}$. aP $<0.05$ vs group I. bP $<$ 0.05 vs group II. $317 \times 293 \mathrm{~mm}(300 \times 300 \mathrm{DPI})$ 


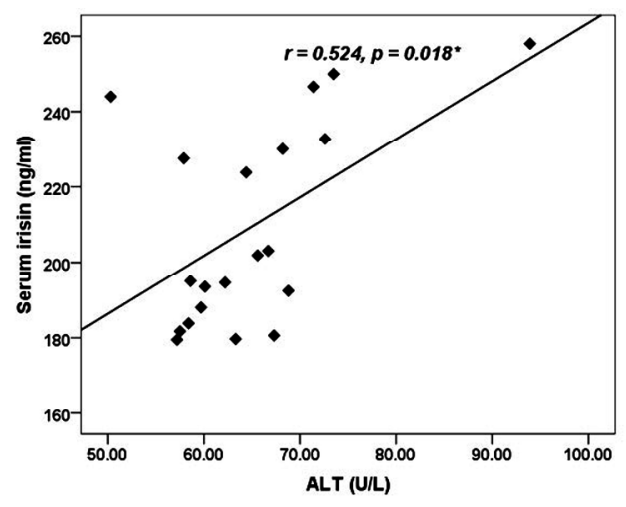

Figure (2) Correlation between serum irisin levels and ALT in patients with metabolic syndrome and elevated liver enzymes. $* p<0.05$ were considered statistically significant. $423 \times 390 \mathrm{~mm}(300 \times 300 \mathrm{DPI})$ 